\title{
The kinematics of Core and Cusp galaxies: comparing HST imaging and integral-field observations
}

\author{
J. Falcón-Barroso ${ }^{1}$, R. Bacon ${ }^{2}$, M. Cappellari ${ }^{3}$, R. L. Davies ${ }^{3}$, \\ P. T. de Zeeuw ${ }^{4}$, E. Emsellem ${ }^{2}$, D. Krajnović ${ }^{3}$, H. Kuntschner ${ }^{5}$, \\ R. M. McDermid ${ }^{4}$, R. F. Peletier ${ }^{6}$, M. Sarzi ${ }^{7}$, and G. van de Ven ${ }^{8}$ \\ 1 European Space and Technology Centre, Keplerlaan 1, 2200 AG Noordwijk, \\ The Netherlands jfalcon@rssd.esa.int \\ 2 Université de Lyon 1, CRAL, Observatoire de Lyon, 9 av. Charles André, 69230 \\ Saint-Genis Laval, France \\ 3 Sub-Department of Astrophysics, University of Oxford, Denys Wilkinson \\ Building, Keble Road, Oxford OX1 3RH, United Kingdom \\ 4 Sterrewacht Leiden, Universiteit Leiden, Postbus 9513, 2300 RA, Leiden, The \\ Netherlands \\ 5 Space Telescope European Coordinating Facility, European Southern \\ Observatory, Karl-Schwarzschild-Str. 2, 85748 Garching, Germany \\ 6 Kapteyn Astronomical Institute, University of Groningen, NL-9700 AV \\ Groningen, The Netherlands \\ 7 Centre for Astrophysics Research, University of Hertfordshire, Hatfield, Herts \\ AL10 9AB, United Kingdom \\ 8 Institute for Advanced Study, Einstein Drive, Princeton, NJ 08540, USA
}

\begin{abstract}
Summary. In this proceeding we look at the relationship between the photometric nuclear properties of early-type galaxies from Hubble Space Telescope imaging and their overall kinematics as observed with the SAURON integral-field spectrograph. We compare the inner slope of their photometric profiles and the Slow/Fast rotator classes, defined by the amplitude of a newly defined $\lambda_{R}$ parameter, to show that slow rotators tend to be more massive systems and display shallower inner profiles and fast rotators steper ones. It is important to remark, however, that there is not a one-to-one relationship between the two photometric and kinematic groups.
\end{abstract}

\section{Introduction}

The study of the galactic nuclei in early-type galaxies has played a fundamental role in our understanding of how galaxies form and evolve. The arrival of high resolution instrumentation with the Hubble Space Telescope (HST) in the early 90's opened a new window into the analysis of the nuclear properties of these systems. One of the major discoveries by HST in this respect is the apparent dichotomy in the nuclear structural properties of these galaxies (see Fig.1). First highlighted by [7] and soon after extended by [10], the core/cusp properties of early-type galaxies have been, and still are, under severe scrutiny by many groups around the world (e.g. $[13,14])$. Recently the 


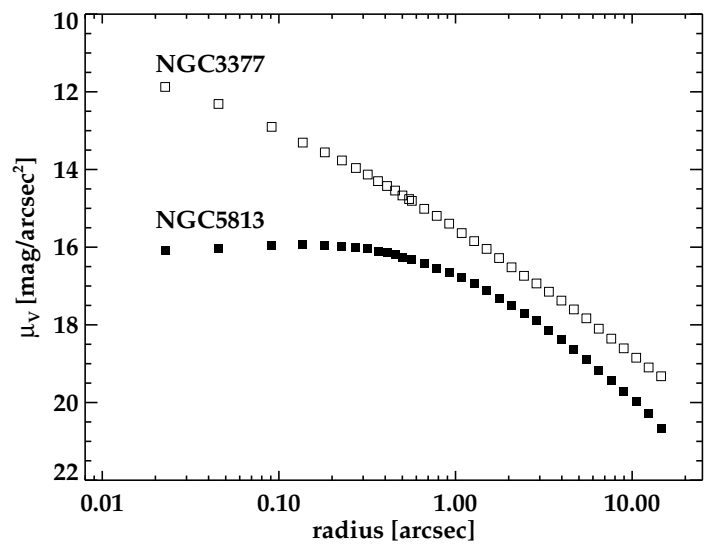

Fig. 1. An illustration of a core and a cuspy nuclear profiles of two galaxies in the SAURON sample from HST V-band observations. Data from [11].

existence of a dichotomy has been questioned by [8] from ACS observations as part of the ACS Virgo Cluster Survey [2] (see also A. Jordán contribution in these proceedings). The topic, however, is far from settled as illustrated by the numerous recent papers debating the existence of the two classes (e.g. see $[9,12]$ and references therein for an in-depth discussion on the subject). Independently of the debate, it is unquestionable that HST is the only current facility in the world that can allow us to revisit this issue.

Almost in parallel to the progress made by HST, ground-based integralfield spectroscopy slowly started to emerge allowing us to look at galaxies in a different new way. With the first observations in 1999, the SAURON project [3] has been one of the pioneers in the exploitation of this technology to study the kinematical properties of galaxies. In this contribution we made use of one of the latest results from our survey to connect the nuclear photometric properties of early-type galaxies with their overall level of rotation. A more detailed analysis of the results presented here is the matter of study in [5].

\section{Core and Cusp galaxies in the SAURON sample}

There are 33 galaxies in the SAURON sample of 48 elliptical and lenticular galaxies with known values for their inner profile slope, which separates them in "core" and "power-law" galaxies $[6,14,13,11]$. From the point of view of their stellar kinematics (see [4]) early-type galaxies appear in two broad flavours, depending on whether they exhibit clear large-scale rotation or not. We measure the level of rotation via a new parameter $\left(\lambda_{R}\right)$ and use it as a basis for a new kinematic classification that separates early-type galaxies into slow and fast rotators in [5]. We have defined the new quantity $\lambda_{R}$ : 

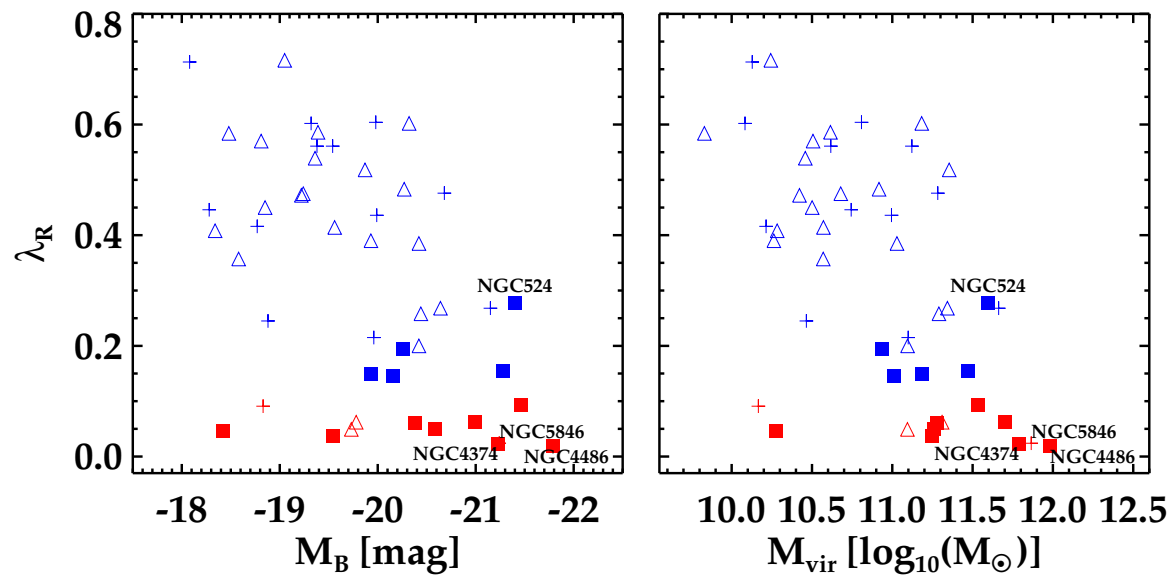

Fig. 2. $\lambda_{R}$ versus absolute magnitude $M_{B}$ (left panel) and virial mass $\mathrm{M}_{v i r}$ (right panel) for the $48 \mathrm{E}$ and $\mathrm{S} 0$ of the SAURON sample. In both panels, symbols correspond to the inner slope classification $[6,14,13,11]$ with power-laws as open triangles, cores as filled squares, and crosses indicating galaxies for which there is no published classification. Slow rotators are coloured in red and fast rotators in blue.

$$
\lambda_{R} \equiv \frac{\langle R|V|\rangle}{\left\langle R \sqrt{V^{2}+\sigma^{2}}\right\rangle},
$$

that measures the amount of specific (projected) angular momentum from the velocity maps. The parameter has been defined such that is insensitive to small features in the maps, and therefore provides a robust measurement of the global rotation. As we go from galaxies with low to high $\lambda_{R}$ values, the overall velocity amplitude naturally tends to increase. More importantly, there seems to be a change in the observed stellar velocity structures.

In the left panel of Fig. 2, we show the distribution of $\lambda_{R}$ as a function of absolute magnitude $M_{B}$. The three slowest rotators (NGC 4486, NGC 4374, NGC 5846) are among the brightest galaxies in our sample with $M_{B}<-21$ mag. Other slow rotators tend to be bright but are spread over a wide range of absolute magnitude. Most fast rotators are fainter than $M_{B}>-20.5 \mathrm{mag}$. In terms of core/cusp distribution, we find that most slow rotators are core galaxies while most fast rotators display power-law profiles. Interestingly there are no core galaxies with $\lambda_{R}>0.3$, although the inclined core galaxy NGC 524 would probably have a very high $\lambda_{R}$ value if seen edge-on. The general behavior that core galaxies have lower $\lambda_{R}$ than cusp galaxies is expected, since both classifications show trends with total luminosity, with brighter members tending to be core galaxies, and lower luminosity ones having power-law profiles [6]. Indeed, all galaxies with $\lambda_{R}>0.3$ have $M_{B}>-20.7$. It is important to notice though that there is not a one-toone correspondance between the different photometric and kinematic groups, since we find both "power-laws" in slow rotators and "cores" in fast rotator. 
One can appreciate in the figure that there is a domain in luminosity and also in mass where both cusp and core galaxies coexist.

In the right panel of Fig. 2, we show the same trends but now as a function of the total mass of the galaxies. We have derived the mass values assuming it approximates to the virial mass derived from the best-fitting $\mathrm{M}_{v i r}-\sigma$ relation presented in [1]. The figure displays a trend with $\lambda_{R}$ such that smaller values are found in more massive galaxies. The three slowest rotators are in the high range of $\mathrm{M}_{v i r}$ with values above $10^{11.5} \mathrm{M}_{\odot}$. There is a clear overlap in mass between fast and slow rotators for $\mathrm{M}_{v i r}$ between $10^{11}$ and $10^{11.5} \mathrm{M}_{\odot}$. However, all slow rotators, have $\mathrm{M}_{\text {vir }}>10^{11} \mathrm{M}_{\odot}$, whereas most fast rotators have $\mathrm{M}_{v i r}<10^{11} \mathrm{M}_{\odot}$, lower masses being reached as the value of $\lambda_{R}$ increases.

The lack of one-to-one relationship between the photometric and kinematic classes is probably highlighting the complex merging histories these galaxies go through in their evolution. Based on the studies showing the incidence of gas in our sample $[15,5]$, it is likely that dissipation is one of the dominant factors in the way galaxies in the different classes evolve. In a forthcoming paper (Falcón-Barroso et al., in preparation) we will combine HST and groung-based imaging with our integral-field observations to extend the analysis presented in this contribution, and to investigate in more detail the links between core/cups and slow/fast rotators in our sample of galaxies.

\section{References}

1. Cappellari M., et al., 2006, MNRAS, 366, 1126

2. Côté P., et al., 2004, ApJS, 153, 223

3. de Zeeuw P. T., et al., 2002, MNRAS, 329, 513

4. Emsellem E., et al., 2004, MNRAS, 352, 721

5. Emsellem E., et al., 2007, MNRAS, 379, 401

6. Faber S. M., et al., 1997, AJ, 114, 1771

7. Ferrarese L., van den Bosch F. C., Ford H. C., Jaffe W., O'Connell R. W., 1994, AJ, 108, 1598

8. Ferrarese L., et al., 2006, ApJS, 164, 334

9. Ferrarese L., et al., 2006, "Black Holes: from Stars to Galaxies - Across the Range of Masses", Proceedings IAU Symposium No. 238, eds. V. Karas \& G. Matt.(astro-ph/0609762)

10. Lauer T. R., et al., 1995, AJ, 110, 2622

11. Lauer T. R., et al., 2005, AJ, 129, 2138

12. Lauer T. R., et al., 2007, ApJ, 664, 226

13. Ravindranath S., Ho L. C., Peng C. Y., Filippenko A. V., Sargent W. L. W., 2001, AJ, 122, 653

14. Rest A., van den Bosch F. C., Jaffe W., Tran H., Tsvetanov Z., Ford H. C., Davies J., Schafer J., 2001, AJ, 121, 2431

15. Sarzi M., et al., 2006, MNRAS, 366, 1151 